\title{
The Booting-Type ADRC of Airborne Photoelectrical Platform
}

\author{
Xiantao Li, ${ }^{1,2}$ Bao Zhang, ${ }^{1}$ Honghai Shen, ${ }^{1}$ and Dapeng Tian ${ }^{1}$ \\ ${ }^{1}$ Changchun Institute of Optics Fine Mechanics and Physics, Chinese Academy of Sciences, No. 3888, Dong-Nan-Hu Road, \\ Changchun 130033, China \\ ${ }^{2}$ Key Laboratory of Airborne Optical Imaging and Measurement, Chinese Academy of Sciences, No. 3888, Dong-Nan-Hu Road, \\ Changchun 130033, China \\ Correspondence should be addressed to Bao Zhang; cleresky@vip.sina.com
}

Received 20 May 2014; Revised 24 August 2014; Accepted 29 September 2014; Published 2 November 2014

Academic Editor: Shihua Li

Copyright (C) 2014 Xiantao Li et al. This is an open access article distributed under the Creative Commons Attribution License, which permits unrestricted use, distribution, and reproduction in any medium, provided the original work is properly cited.

Customary disturbance rejection in the photoelectrical platform is "passive," which makes it difficult to further improve the isolation degree owning to the strict restriction of mechanical resonance frequency. In this paper, a booting-type ADRC is proposed, whose disturbance estimation process is guided by target value to reduce the overshoot and lag in the estimated value of disturbance. All kinds of disturbance in the system are modeled in a unified way by using the equivalent disturbance voltage to avoid the complex modeling process. Based on the simplified model, extended state observer (ESO) is designed to realize a real-time estimation of the disturbance. Then, the disturbance compensation is added to generate the final control value by combining the customary square lead-lag controller. Experiments are implemented to test the proposed control strategy by mounting the photoelectrical platform on a flight simulator and generating a motion perturbation. Compared with the case of only traditional lead-lag controller, the isolation degree of disturbance is enhanced obviously. And the experiments also illustrate strong robustness of ADRC.

\section{Introduction}

Photoelectrical stabilized platform is widely used in aeronautic and astronautic imaging systems [1-3]. This platform effectively isolates the disturbance of angular motion of the aerial vehicle and guarantees the direction of the axis-ofsight stable in the inertial space [4]. With the photoelectrical platform, the quality of imaging is improved.

The photoelectrical platform is disturbed by the motion of the aerial vehicle through the friction in each axis. This friction disturbance directly influences the direction of optical lens, which degrades the quality of imaging [5, 6]. Therefore, the improvement of isolation degree against the disturbance is the key issue to enhance the quality of imaging in the photoelectrical platform. However, until now, normal approach of improving the isolation degree follows the idea of "passive disturbance rejection." The influence of disturbance decreases by enlarging the gain of controller in low frequency and designing a controller to construct a high-order system. However, because of the resonance in the mechanism, large gain in the usual feedback controller is difficult to achieve. To overcome this problem, there are two feasible lines. One is increasing the mechanical design, for example, fast steering mirror (FSM) that has small inertial mass and resonance designed [7]. The other is employing advanced control algorithm. Modern control theories, such as the optimum control, adaptive control [8], come into force on improving the isolation degree of the photoelectrical platform. However, a lot of past designs of the high performance control algorithm are complex and not easy to realize in practical engineering.

Active disturbance rejection controller (ADRC) $[9,10]$ is a novel technique founded on the idea of "active disturbance rejection." This approach extracts the information of the disturbance from the signals of the input and output of a plant. The external disturbance and internal uncertainty are modeled in a unified way. Then they are estimated by the ESO and compensated. By combining the disturbance rejection with traditional feedback controller, the disturbance is rejected under both "active" and "passive" control effects. 


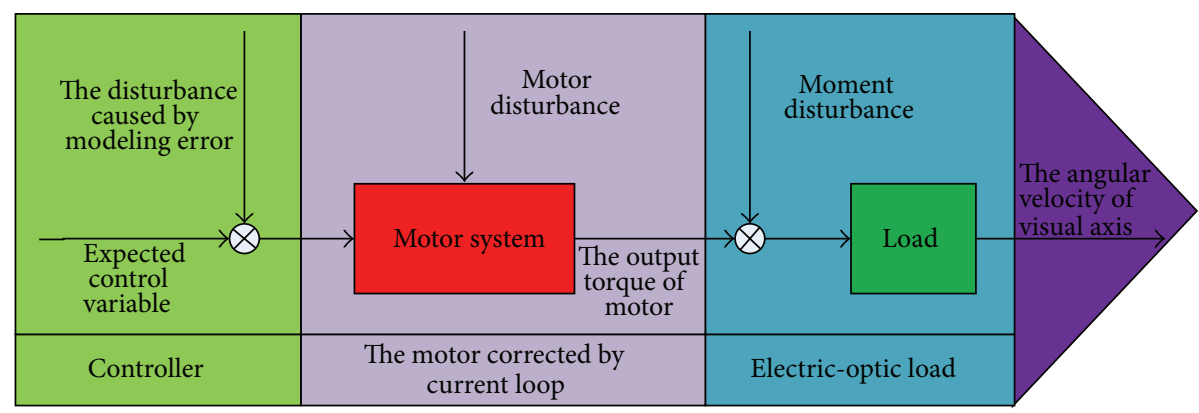

FIGURE 1: The principle of the disturbance in system.

However, the overshoot and lag in the estimated value of disturbance degrade the performance of the traditional active disturbance rejection controller (ADRC) and even lead to instability. To solve this problem, the booting-type ADRC whose disturbance estimation process is guided by target value is proposed. In this controller, the total control value is generated by combining the active disturbance rejection with the square lead-lag compensation. Experimental results prove that the booting-type ADRC effectively improves the disturbance observation. The disturbance summation is estimated and compensated actively, which improves the isolation degree of the photoelectrical platform compared with traditional passive compensation.

\section{Modeling and Simplification of the Airborne Photoelectrical Platform}

The 2-axis airborne photoelectrical platform is investigated in this paper, which was driven by torque motors. The angular velocity of each axis in the inertial space is measured by a high accuracy gyro. The objective of the system is to stabilize the two axes in the inertial space, which means the angular velocity of each axis in the inertial space is zero even though there is attitude disturbance caused by the aerial vehicle.

The pitching axis is perpendicular to the yawing axis. Therefore, the influence of the coupling between the two axes is insignificant. This paper examines the issue of designing ADRC in yawing axis for example.

The relationship between the control voltage $u_{a}$ and the velocity response $\omega_{M}$ of the controlled plant and the torque motor driven system is usually described by the model shown in Figure 1.

In Figure 1, the electric current loop is used in the motor system to reject the electronic noise, back emf, and electromagnetic interference. On this basis, the motor system will output the torque according to the control voltage. Thus, the transfer function of the motor system considering the slight bias is

$$
T_{M}=K_{T} u+T_{M D}
$$

where $T_{M}$ is the output torque of the motor system, $K_{T}$ is the proportional coefficient determined according to the design of the electric current loop, and $T_{M D}$ is the slight bias of output torque. The kinematics equation on the $x$-axis of the airborne photoelectrical platform is

$$
\begin{aligned}
T_{M} & =K_{T} u \\
& =T_{L}+J_{\mathrm{Ges}} \frac{d \omega_{M}}{d t},
\end{aligned}
$$

where $\omega_{M}$ is the angular velocity on the yawing axis. $J_{\mathrm{Ges}}$ is the moment of inertia of the airborne photoelectrical platform on the yawing axis. $T_{L}$ is the disturbance torque including friction torque, mass unbalance torque, and moment of wind resistance. From (1) and (2), there are

$$
\begin{aligned}
K_{T} u+T_{M D}=T_{L}+J_{\mathrm{Ges}} \frac{d \omega_{M}}{d t} \\
\Longrightarrow \frac{d \omega_{M}}{d t}=\frac{1}{J_{\mathrm{Ges}}}\left(K_{T} u+T_{M D}-T_{L}\right) \\
=\frac{K_{T}}{J_{\mathrm{Ges}}}\left(u+\frac{1}{K_{T}} T_{M D}-\frac{1}{K_{T}} T_{L}\right) .
\end{aligned}
$$

Using Laplace transformation, (4) is rewritten to

$$
\omega_{M}(s)=\frac{K_{T}}{J_{\mathrm{Ges}} s}\left(u(s)+\frac{1}{K_{T}} T_{M D}(s)-\frac{1}{K_{T}} T_{L}(s)\right) .
$$

Suppose $k=K_{T} / J_{\mathrm{Ges}}$, (5) is rewritten to

$$
\omega_{M}(s)=\frac{k}{s}\left(u(s)+\frac{1}{K_{T}} T_{M D}(s)-\frac{1}{K_{T}} T_{L}(s)\right),
$$

where $k$ is the model parameter whose concrete value is determined by practical system characteristics. But its exact value is almost impossible to be obtained in actual engineering. Suppose $k^{\prime}$ is the parameter of nominal model. It is inevitable that there will be error between $k^{\prime}$ and $k$. The error is called the quiet modeling error. So, the airborne photoelectrical platform could not achieve the expectation characteristic 
by using the controller designed according to the nominal model. In order to correct the error, (6) is rewritten as

$$
\begin{aligned}
\omega_{M}(s)= & \frac{k^{\prime}}{s} \cdot \frac{k}{k^{\prime}}\left(u(s)+\frac{1}{K_{T}} T_{M D}(s)-\frac{1}{K_{T}} T_{L}(s)\right) \\
= & \frac{k^{\prime}}{s}\left(u(s)+\frac{\left(k-k^{\prime}\right)}{k^{\prime}} u(s)+\frac{k}{K_{T} k^{\prime}} T_{M D}(s)\right. \\
& \left.\quad-\frac{k}{K_{T} k^{\prime}} T_{L}(s)\right) \\
= & \frac{k^{\prime}}{s} \cdot u(s)+\frac{k^{\prime}}{s} \\
& \times\left(\frac{\left(k-k^{\prime}\right)}{k^{\prime}} u(s)+\frac{k}{K_{T} k^{\prime}} T_{M D}(s)-\frac{k}{K_{T} k^{\prime}} T_{L}(s)\right),
\end{aligned}
$$

where $\left(k^{\prime} / s\right) \cdot u(s)$ is the expectation characteristic, $(k / s) \cdot u(s)$ is the actual characteristic, $\left(k^{\prime} / s\right) \cdot\left(\left(k-k^{\prime}\right) / k^{\prime}\right) u(s)$ is the bias caused by modeling error, $\left(k^{\prime} / s\right) \cdot\left(k / K_{T} k^{\prime}\right) T_{M D}$ is the bias caused by motor interference, and $\left(k^{\prime} / s\right) \cdot\left(-\left(k / K_{T} k^{\prime}\right) T_{L}(s)\right)$ is the bias caused by the disturbance torque. Obviously, they have the same form. And they all make control voltage deviated from the expected value. Now let $d(s)=((k-$ $\left.\left.k^{\prime}\right) / k^{\prime}\right) u(s)+\left(k / K_{T} k^{\prime}\right) T_{M D}-\left(k / K_{T} k^{\prime}\right) T_{L}(s)$, where $d(s)$ is called the equivalent disturbance voltage. It means that if $d(s)$ and all the disturbances in the system have the same jamming effect; the various disturbances in the system could be modeled in a unified way by using the equivalent disturbance voltage $d(s)$. Then the transfer function of the airborne photoelectrical platform is simplified as in the following equation:

$$
\omega_{M}(s)=\frac{k^{\prime}}{s}(u(s)+d(s)) .
$$

The modeling method avoids the complex modeling process for the various disturbances in the system. Besides, this method is very suitable for the active disturbance rejection controller as described below.

\section{The Strategy of Disturbance Rejection}

According to (8), the principle of speed loop in the system is illustrated as in Figure 2, where $R(s)$ is the expected angular velocity to inertial space, $G(s)$ is the transfer function of the controller, $d(s)$ is the equivalent disturbance voltage, and $P(s)$ represents the controlled system, $C(s)$ is the actual angular velocity. The transfer function of the speed loop is expressed as

$$
C(s)=\frac{G(s) P(s)}{1+G(s) P(s)} R(s)+\frac{P(s)}{1+G(s) P(s)} d(s) .
$$

The error transfer function is shown as

$$
E(s)=\frac{1}{1+G(s) P(s)} R(s)-\frac{P(s)}{1+G(s) P(s)} d(s),
$$

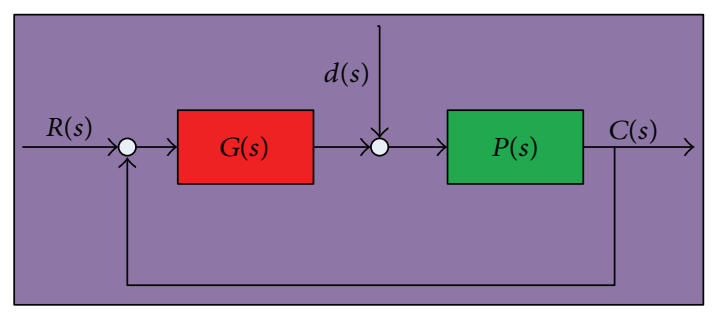

FIgURE 2: The principle of the speed loop.

where $(1 /(1+G(s) P(s))) R(s)$ is steady-state error and $(p(s) /(1+G(s) P(s))) d(s)$ is influence of the system output of the equivalent disturbance voltage for the system output. The steady-state error could be ignored because $|1+G(s) P(s)|$ is much larger than 1 . Therefore, the disturbance is the primary factors affecting the stability of the system. $(p(s) /(1+$ $G(s) P(s)) d(s)$ can be reduced in two ways, by designing the controller $G(s)$ to increase $|1+G(s) P(s)|$ in the frequency range of the disturbance and by using the disturbance's estimated value to reduce $d(s)$ directly.

The former one is the traditional method based on the idea of "passive disturbance rejection" and its ability of disturbance rejection is difficult to be improved further owning to the restriction of mechanical resonance frequency, such as the PI controller and the square lead-lag controller. Compared with the PI controller, the isolation degree of disturbance by using the square lead-lag controller is higher. So the square lead-lag controller is employed to be the contrast experiment in this paper. The analysis is as follows.

The transfer function of the square lead-lag controller is

$$
G_{\text {square lead-lag }}=k_{p} \frac{\left(\left(1 / T_{1}\right) s+1\right)^{2}}{\left(\left(1 / T_{2}\right) s+1\right)^{2}} .
$$

The transfer function of the PI controller is

$$
G_{\mathrm{PI}}=k_{p}\left(1+\frac{1}{T_{I} s}\right) \text {. }
$$

The two controllers are used to control the same system, respectively. The transfer function of the system is $k / s$. The two transfer curves of their open-loop characteristics are illustrated in Figure 3.

For the purposes of comparison and the restriction of mechanical resonance, the two transfer curves have the same middle-frequency. According to Figure 3, compared with the blue curve, the red curve is obviously higher in the range of CA. According to the previous analysis, the ability of disturbance rejection by using the square lead-lag controller is obviously higher compared with using the PI controller.

The latter method is a novel technique based on the idea of "active disturbance rejection." The equivalent disturbance voltage $d(s)$ is estimated by disturbance observer, and then it is compensated by the disturbance's estimated value $d^{\prime}(s)$. Its principle is shown as

$$
\frac{P(s)}{1+G(s) P(s)} d(s) \longrightarrow \frac{P(s)}{1+G(s) P(s)}\left(d(s)-d^{\prime}(s)\right) .
$$






FIgURE 3: The transfer curves of the open-loop characteristics.



FIgURE 4: The principle of ADRC.

Obviously, the disturbance $d(s)$ is rejected under both "active" and "passive" control effects. The isolation degree of disturbance is improved further compared with the traditional method. DOB, SPID, and ADRC all are part of this method. The difference between them is the method for their obtaining the disturbance's estimated value $d^{\prime}(s)$. ADRC is employed in this paper.

\section{Design of the Booting-Type ADRC}

The ADRC is a preferable technique based on the idea of "active" disturbance rejection. The core idea is actively estimating and compensating the disturbance summation by seeing the disturbance summation as a system state and designing the extended state observer. The principle of ADRC is illustrated in Figure 4.

4.1. Design of ESO [11]. The ESO is designed in the state space $[12,13]$. Therefore, the controlled plant $(8)$ is rewritten to the expression in state space (14). Consider

$$
\begin{gathered}
\dot{x}_{1}=k^{\prime} d+k^{\prime} u, \\
y=x_{1} .
\end{gathered}
$$

Suppose $x_{2}=k^{\prime} d$ and the derivation of $x_{2}$ is $\omega(t) . x_{2}$ is the extended state. Then, the first-order system (14) is rewritten to the second-order system (15). Consider

$$
\begin{gathered}
\dot{x}_{1}=x_{2}+k^{\prime} u, \\
\dot{x}_{2}=\omega(t), \\
y=x_{1} .
\end{gathered}
$$

Then, second-order ESO is designed for above second-order system to estimate the equivalent disturbance voltage by making $d^{\prime} \rightarrow d$. $d^{\prime}$ is the estimated value of the disturbance $d$. The ESO is designed as

$$
\begin{gathered}
e_{1}=z_{1}-y, \\
\dot{z}_{1}=z_{2}-\beta_{01} e_{1}+k^{\prime} u, \\
\dot{z}_{2}=-\beta_{02} \mathrm{fal}\left(e_{1}, \frac{1}{2}, \delta\right), \\
d^{\prime}=\frac{z_{2}}{k^{\prime}},
\end{gathered}
$$

where $\operatorname{fal}(e, \alpha, \delta)$ is composed of a linear function and a nonlinear function as

$$
\mathrm{fal}(e, \alpha, \delta)= \begin{cases}\frac{e}{\delta^{\alpha-1}}, & |e| \leq \delta \\ |e|^{\alpha} \operatorname{sign}(e), & |e|>\delta\end{cases}
$$

The parameter $\delta$ determines the working interval of the linear function in $\operatorname{fal}(e, \alpha, \delta)$. The linear function is convergent, which avoids the oscillation when the argument enters the interval near the equilibrium point of the function. The nonlinear function has high speed of convergence, which enhances the dynamic performance of the ESO. The parameter $\delta$ should be chosen greater than the noise of velocity measurement. The peak value of the noise in the gyro of the system in this paper is $0.435^{\circ} / \mathrm{s}$. In the experiments of this paper, the parameter is chosen as $\delta=0.6^{\circ}$ s. The parameters $\beta_{01}$ and $\beta_{02}$ are designed according to the approach of [14] as $\beta_{01}=2 \omega, \beta_{02}=\omega^{2}$. In the experiments of this work, the parameter of the ESO is selected as $\omega=200$.

In order to analyze the measurement accuracy of the extended state observer in the actual system, the experimental device is simplified as follows: except gyroscope and optical shaft encoder, all the electric-optic load in the system is removed. In this way, the disturbance summation is mainly composed of friction torque because of the mass unbalance torque and wire disturbance is avoided easily in the simplified system. The simplified experimental device is shown in Figure 5.

The desired angular velocity is zero when the flight simulation swings the photoelectrical platform. The amplification and frequency of the swinging are $1^{\circ}$ and $1 \mathrm{~Hz}$, respectively. The disturbance summation observed by ESO is illustrated in Figure 6.

As known of the friction torque, its direction is opposite to the relative motion tendency of the interface. Therefore, as showed in Figure 6, the lag time of the disturbance's estimated 


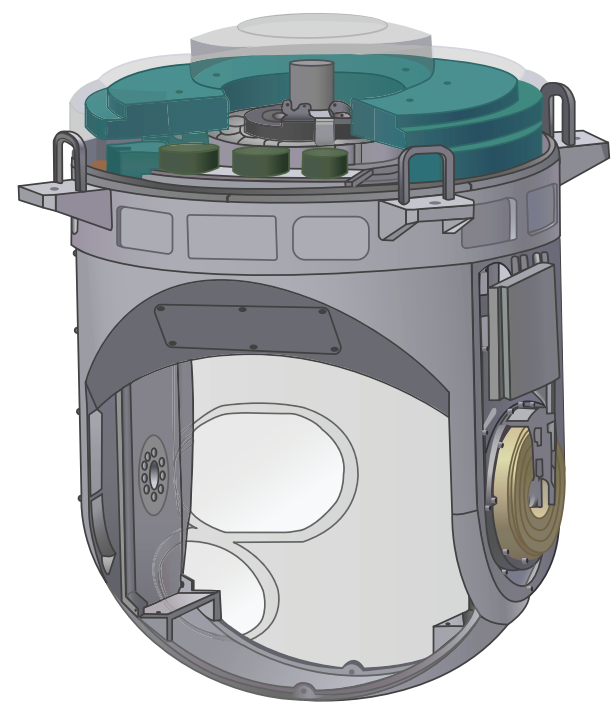

Figure 5: The airborne photoelectrical platform without electricoptic load.

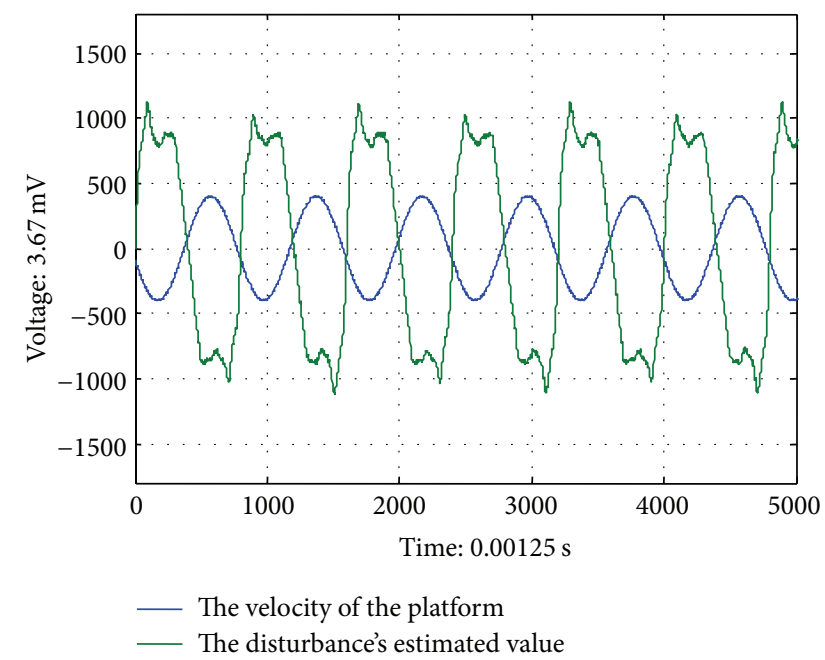

FIGURE 6: The relationship between disturbance's estimated value and velocity of the platform with traditional ESO.

value is approximately $0.025 \mathrm{~s}$. Beyond that, the overshoot of the disturbance's estimated value is very serious. This is because the disturbance changes too fast at the beginning of the relative motion tendency of the interface changes. This phenomenon is the same as step response. It is well known that the phase lag and overshoot degrade the performance of disturbance rejection and even lead to instability. Thus, how to reduce the lag and overshoot of the estimated value is the key factor in improving the performance of ADRC. However, it is hard to solve the problem only by adjusting the parameters $\beta_{01}$ and $\beta_{02}$.

To solve this problem, the booting-type ESO is proposed. It is designed according to the main features of the disturbance in the airborne photoelectrical platform. And its perturbation observation not only rely on adjusting the parameters $\beta_{01}$ and $\beta_{02}$ but also rely on the guide of

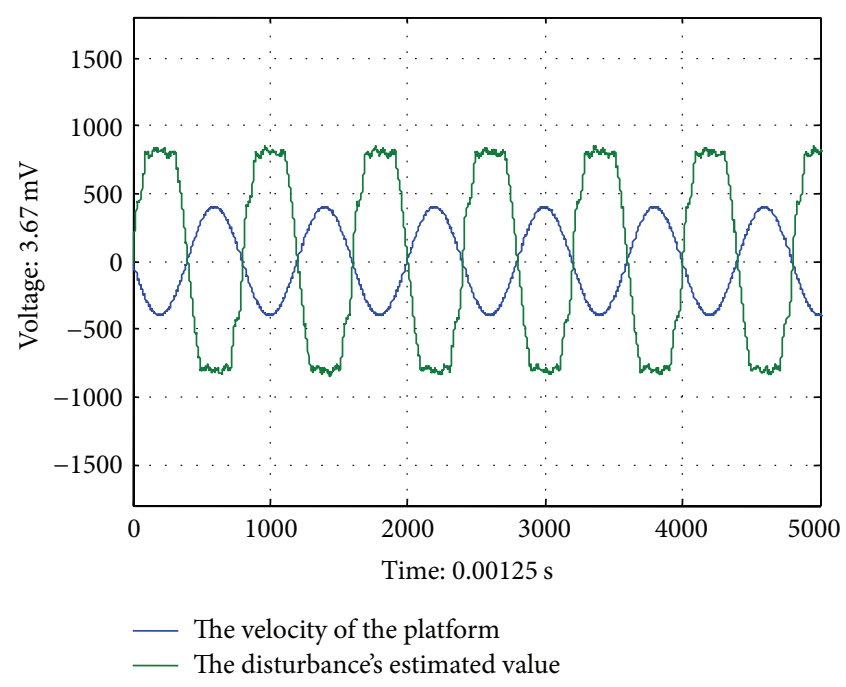

FIGURE 7: The relationship between disturbance's estimated value and velocity of the platform with booting-type ESO.

the equivalent disturbance voltage $d$. Because $d$ is mainly composed of dynamic friction torque, the dynamic friction torque is chosen to be the target value. The value needs not be as pretty accurate as modeling of friction. It only needs to reduce the estimation error at the beginning of the relative motion tendency of the interface changes. The simplest method is to replace the value of $d^{\prime}$ with the friction at this time. The booting-type ESO is intended to

$$
\begin{gathered}
e_{1}=z_{1}-y, \\
\dot{z}_{1}=z_{2}-\beta_{01} e_{1}+k^{\prime} u, \\
\dot{z}_{2}=-\beta_{02} \mathrm{fal}\left(e_{1}, \frac{1}{2}, \delta\right)+f\left(z_{2}, d_{\text {Target }}, \text { dir }\right), \\
d^{\prime}=\frac{z_{2}}{k^{\prime}},
\end{gathered}
$$

where $d_{\text {Target }}$ is the target value. Its value is determined according to the features of disturbance in debugging progress. dir is the relative motion tendency of the interface. $f\left(z_{2}, d_{\text {Target}}\right.$, dir $)$ is a nonlinear function. It can make the bias between the estimated value of the disturbance and the real disturbance which will not be too large at the beginning of the relative motion tendency of the interface changes. This is the major innovative point in this work. The disturbance summation observed by booting-type ESO is illustrated in Figure 7.

Obviously, the phase lag and overshoot of the disturbance's estimated value are reduced greatly.

4.2. Design of Dynamic Linear Compensation. Depending on the estimated value of the equivalent disturbance voltage $d^{\prime}$, the control value $u$ is corrected to $u=u_{0}-d^{\prime}$. Here, $u_{0}$ is the output of the square lead-lag controller. $d^{\prime}$ is the compensation of the disturbance summation. By substituting 
the designed $u$ with active disturbance compensation into (8), the system is rewritten to

$$
\begin{gathered}
\dot{x}_{1}=k^{\prime}\left(u_{0}+d-d^{\prime}\right), \\
y=x_{1} \\
\Longrightarrow \dot{x}_{1}=k^{\prime} u_{0}+k^{\prime}\left(d-d^{\prime}\right), \\
y=x_{1} .
\end{gathered}
$$

Therefore, the active rejection of disturbance is achieved because the booting-type ESO has the convergence $d^{\prime} \rightarrow d$, $d-d^{\prime} \rightarrow 0$.

4.3. The Robustness Analysis of the Booting-Type ADRC. The properties of the airborne photoelectrical platform and sensor will inevitably change because the working environment is complex. Therefore, the controller which is developed based on the nominal model is no longer completely applicable, resulting in the decline of system performance. Robustness is a measure of the stability of the system in the condition of parameter perturbation.

In this paper, the complex motor drive circuit of the airborne photoelectrical platform is simplified to a firstorder model by an electric current loop control. So, $k^{\prime}$ is the only varying parameter which follows the change in the working environment. Suppose $\Delta$ is the variable quantity of the model parameter $k^{\prime}$. In this condition, the system (8) can be rewritten as

$$
\begin{gathered}
\dot{x}_{1}=\left(k^{\prime}+\Delta\right)(u+d), \\
y=x_{1} .
\end{gathered}
$$

When the square lead-lag controller is used separately in the system, the system (20) is rewritten to

$$
\begin{gathered}
\dot{x}_{1}=k^{\prime}\left(u_{0}+\left(d+\frac{\Delta}{k^{\prime}}\left(u_{0}+d\right)\right)\right) \\
y=x_{1},
\end{gathered}
$$

where $\left(\Delta / k^{\prime}\right)\left(u_{0}+d\right)$ is caused by the changing of the model parameter. It will lead to the error of control voltage caused by the changing of the model parameter. According to the concept of disturbance summation in ADRC, $\left(\Delta / k^{\prime}\right)\left(u_{0}+d\right)$ is considered as a part of the equivalent disturbance voltage to be estimated by the booting-type ESO designed when model parameter is $k^{\prime}$. In an ideal world, the booting-type ESO has the convergence $d^{\prime} \rightarrow d+\left(\Delta / k^{\prime}\right)\left(u_{0}+d\right)$. Then the estimated value $d^{\prime}$ is fed into the changed system (9); the system is rewritten to

$$
\begin{aligned}
\dot{x}_{1} & =\left(k^{\prime}+\Delta\right)\left(u_{0}+d-\left(d+\frac{\Delta}{k^{\prime}}\left(u_{0}+d\right)\right)\right) \\
& =\left(k^{\prime}+\Delta\right)\left(u_{0}-\frac{\Delta}{k^{\prime}}\left(u_{0}+d\right)\right) \\
& =k^{\prime} \frac{\left(k^{\prime}+\Delta\right)}{k^{\prime}}\left(u_{0}-\frac{\Delta}{k^{\prime}}\left(u_{0}+d\right)\right)
\end{aligned}
$$

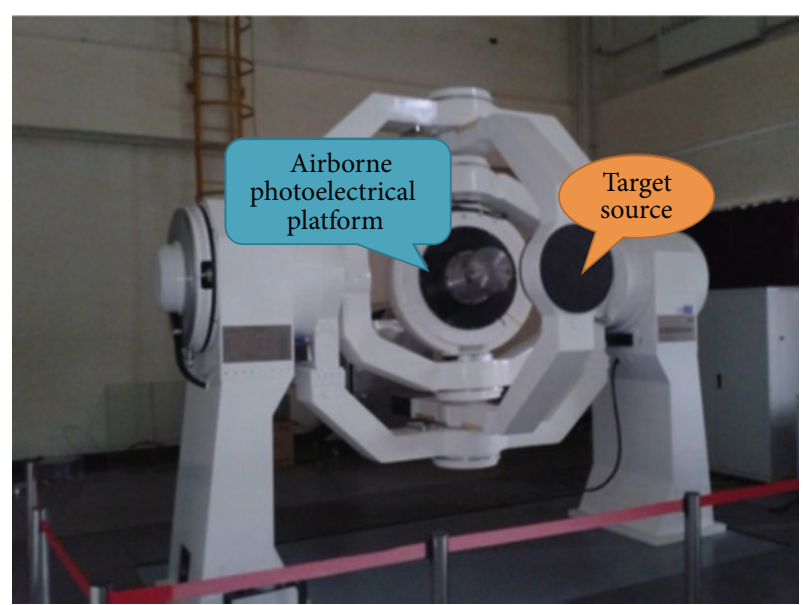

FIgURE 8: The five-axis flight simulator.

$$
\begin{aligned}
& =k^{\prime}\left(\frac{\left(k^{\prime}+\Delta\right)}{k^{\prime}} \cdot u_{0}-\frac{\left(k^{\prime}+\Delta\right)}{k^{\prime}} \cdot \frac{\Delta}{k^{\prime}}\left(u_{0}+d\right)\right) \\
& =k^{\prime}\left(u_{0}+\frac{\Delta}{k^{\prime}} u_{0}-\frac{\Delta k^{\prime}}{\left(k^{\prime}\right)^{2}} u_{0}-\frac{\Delta^{2}}{\left(k^{\prime}\right)^{2}} u_{0}-\frac{\Delta k^{\prime}}{\left(k^{\prime}\right)^{2}} d-\frac{\Delta^{2}}{\left(k^{\prime}\right)^{2}} d\right) \\
& =k^{\prime}\left(u_{0}-\frac{\Delta^{2}}{\left(k^{\prime}\right)^{2}} u_{0}-\frac{\Delta k^{\prime}}{\left(k^{\prime}\right)^{2}} d-\frac{\Delta^{2}}{\left(k^{\prime}\right)^{2}} d\right) \\
& =k^{\prime}\left(u_{0}-\frac{\Delta}{k^{\prime}}\left(\frac{\Delta}{k^{\prime}} u_{0}+d+\frac{\Delta}{k^{\prime}} d\right)\right) \\
& =k^{\prime}\left(u_{0}-\frac{\Delta}{k^{\prime}}\left(d+\frac{\Delta}{k^{\prime}}\left(u_{0}+d\right)\right)\right) .
\end{aligned}
$$

Compared with (21), $d+\left(\Delta / k^{\prime}\right)\left(u_{0}+d\right)$ is changed into $\left(-\left(\Delta / k^{\prime}\right)\left(d+\left(\Delta / k^{\prime}\right)\left(u_{0}+d\right)\right)\right)$. Because the value of $\Delta / k^{\prime}$ is less than 0.2 in engineering practice, the amplitude of $d$ and $\left(\Delta / k^{\prime}\right)\left(u_{0}+d\right)$ are all greatly reduced. Adding it all up, the booting-type ADRC is effective and robust against the changing of the model.

\section{Experiments on the Stabilization of the Axis-of-Sight}

To verify the improvement of the isolation degree by ADRC compared with customary square lead-lag control, the experiments are performed by mounting the photoelectrical platform on a flight simulator. The flight simulator is given in Figure 8 . The experiments of velocity stabilization, target tracking, and verification of robustness are implemented.

5.1. Experiment of Velocity Stabilization. The desired angular velocity is zero when the flight simulation swings the photoelectrical platform. The amplification and frequency of the swinging are $1^{\circ}$ and $1.5 \mathrm{~Hz}$, respectively. The real angular velocity is measured by a gyro. The experimental results are illustrated in Figures 9(a) and 9(b). 




(a)

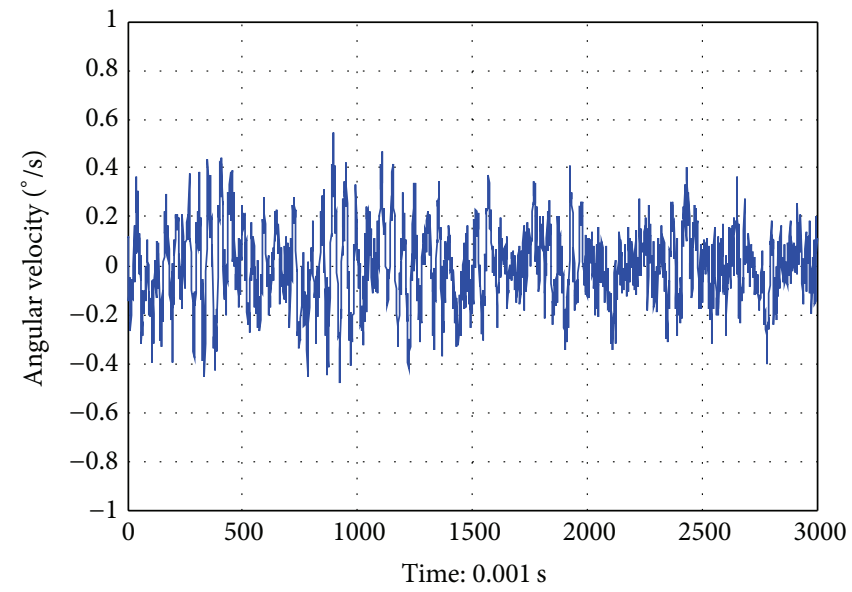

(b)

FIGURE 9: (a) Square lag-lead controller response to $1.5 \mathrm{~Hz}$ disturbance. (b) ADRC response to $1.5 \mathrm{~Hz}$ disturbance.

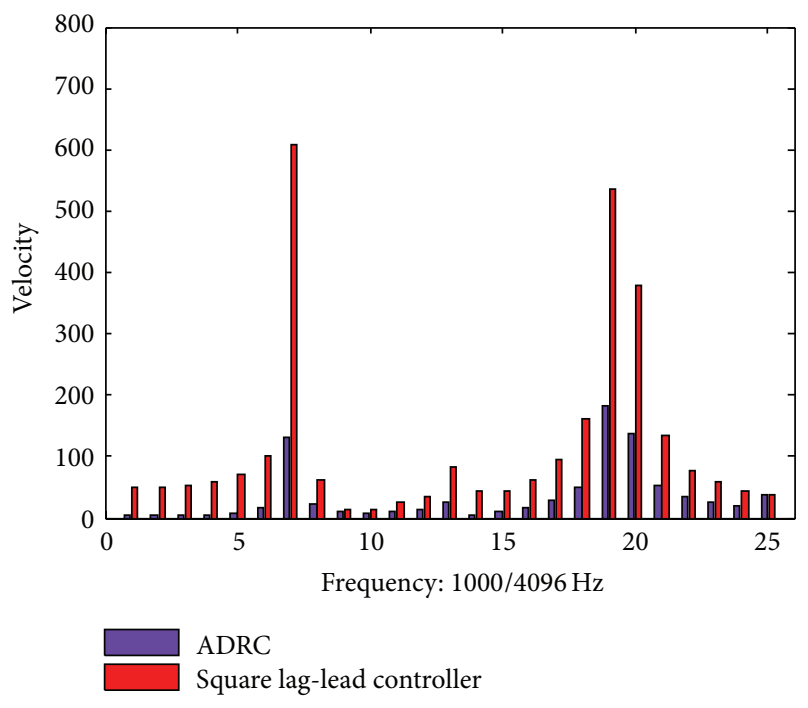

FIGURE 10: Fourier transform of rate when the photoelectrical stabilized platform is disturbed by $1.5 \mathrm{~Hz}$ disturbance.

According to Figure 9, compared with only feedback control using square lead-lag controller, the error of angular velocity stabilization is obviously smaller using the ADRC. Most of the error is under the level of $0.5^{\circ} / \mathrm{s}$. The peak value of the noise of the gyro is $0.435^{\circ} / \mathrm{s}$. Therefore, the result using ADRC is closed to the limitation of the system.

Frequency spectrum analysis is performed for the results in Figure 8 using Fourier transform. The results are presented in Figure 10. According to this figure, the oscillation of $1.5 \mathrm{~Hz}$ using ADRC is just $1 / 5$ of the situation with only feedback control by the square lead-lag controller. It means that the isolation degree of the system is improved by $13.98 \mathrm{~dB}$ in this frequency. Besides the velocity perturbation from the aerial vehicle, the ADRC is also effective in the disturbances in other frequencies caused by the friction and unbalanced moments.
TABLE 1: The improvement of disturbance isolation degree by using ADRC compared with square lead-lag controller.

\begin{tabular}{lc}
\hline Frequency $[\mathrm{Hz}]$ & Improvement of isolation degree $[\mathrm{dB}]$ \\
\hline 0.1 & 7.12 \\
0.5 & 11.28 \\
1.0 & 12.83 \\
1.5 & 13.98 \\
2.0 & 12.78 \\
2.5 & 12.94 \\
\hline
\end{tabular}

To illustrate the improvement of isolation degree in wider bandwidth, the performance of the system is also tested by making the flight simulator generate a sinusoidal angular perturbation. The amplification of the perturbation is $1^{\circ}$. The frequency is selected in each $0.5 \mathrm{~Hz}$ between $0.1 \mathrm{~Hz}$ and $2.5 \mathrm{~Hz}$. The improvement of isolation degree using ADRC is shown in Table 1. Compared with only square lead-lag controller, the isolation degree is effectively improved in each frequency. In higher frequency, the performance of passive disturbance rejection by traditional controller is degraded. The advantage of active disturbance rejection is more noticeable in higher frequency.

5.2. Experiment of Target Tracking. In this experiment, the imaging system in the photoelectrical platform tracks a point target in the infinite place. The angular error between the axis-of-sight and the target is monitored by a video tracker. The photoelectrical platform is also mounted in the flight simulator. A sinusoidal angular perturbation with the amplification of $1^{\circ}$ is generated.

Figures 11(a) and 11(b) illustrate the angular error of the $1.5 \mathrm{~Hz}$ angular perturbation. Depending on this figure, the amplification of the oscillation with ADRC is obviously smaller than the situation of only using traditional square lead-lag controller. The error using ADRC is about 


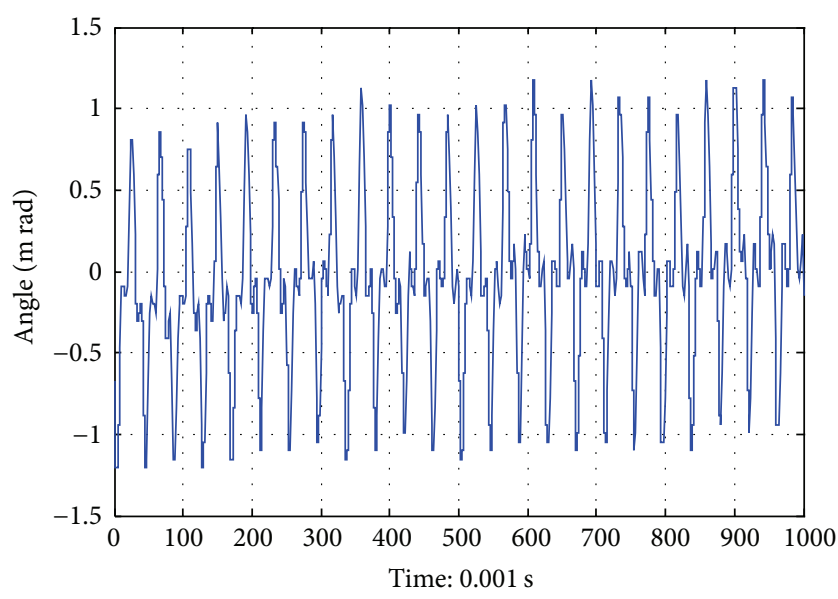

(a)

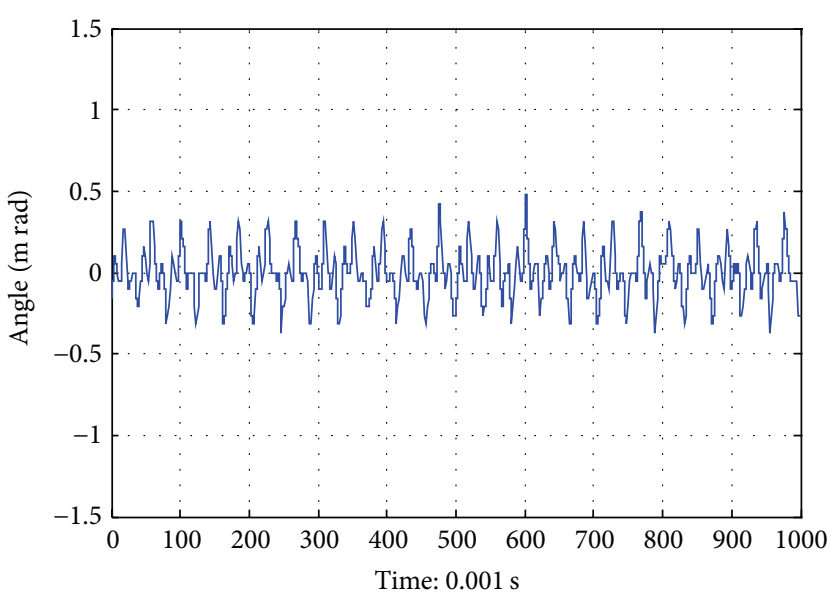

(b)

FIGURE 11: (a) The range of visual axis sloshing when the photoelectrical stabilized platform by using square lag-lead controller is disturbed by $1.5 \mathrm{~Hz}$ disturbance. (b) The range of visual axis sloshing when the photoelectrical stabilized platform by using ADRC is disturbed by $1.5 \mathrm{~Hz}$ disturbance.

TABLE 2: The range of visual axis sloshing when the photoelectrical stabilized platform is in tracking state.

\begin{tabular}{lcc}
\hline Frequency $[\mathrm{Hz}]$ & $\begin{array}{c}\text { Using ADRC } \\
{[\mathrm{mrad}]}\end{array}$ & $\begin{array}{c}\text { Using traditional lead-lag } \\
\text { controller [mrad] }\end{array}$ \\
\hline 0.1 & \pm 0.053 & \pm 0.106 \\
0.5 & \pm 0.165 & \pm 0.636 \\
1.0 & \pm 0.212 & \pm 0.954 \\
1.5 & \pm 0.318 & \pm 1.113 \\
2.0 & \pm 0.318 & \pm 1.219 \\
2.5 & \pm 0.318 & \pm 1.219 \\
\hline
\end{tabular}

$\pm 0.318 \mathrm{mrad}$ which is only $1 / 3$ of the situation using traditional controller.

We also test the error with the angular perturbation in each $0.5 \mathrm{~Hz}$ frequency between $0.1 \mathrm{~Hz}$ and $2.5 \mathrm{~Hz}$. The error in each frequency is shown in Table 2. According to the results, using ADRC makes the imaging system aim at the target with a smaller error in the presence of disturbance.

5.3. Experiment of Controller Robustness. To verify the robustness of the ADRC, the mass of the load on the platform is changed to make the parameter $k$ in (8) changed within the range of $\Delta= \pm 15 \%$. With this model unmatching, the experiments in 5.1 and 5.2 are carried out again. The results are listed in Table 3.

Obviously, the ADRC tolerates the parameter changing within $\pm 15 \%$ in the model. The isolation degree of the platform is still improved compared with only traditional feedback controller. The improved isolation degree meets the requirement in engineering. Therefore, the booting-type ADRC is efficient and robust against load changing.
TABLE 3: The improvement of isolation degree and the range of visual axis sloshing using ADRC when $\Delta=-15 \%$ and $\Delta=15 \%$.

\begin{tabular}{lcc}
\hline \multirow{2}{*}{ Frequency $[\mathrm{Hz}]$} & \multicolumn{2}{c}{$\begin{array}{l}\text { Improvement of isolation degree } \\
{[\mathrm{dB}] / \text { range of visual axis }[\mathrm{mrad}]}\end{array}$} \\
& $\Delta=-15 \%$ & $\Delta=15 \%$ \\
\hline 0.1 & $6.38 / \pm 0.053$ & $6.15 / \pm 0.053$ \\
0.5 & $9.35 / \pm 0.212$ & $10.12 / \pm 0.265$ \\
1.0 & $10.72 / \pm 0.318$ & $10.63 / \pm 0.265$ \\
1.5 & $10.85 / \pm 0.371$ & $10.88 / \pm 0.371$ \\
2.0 & $10.83 / \pm 0.371$ & $10.75 / \pm 0.424$ \\
2.5 & $10.76 / \pm 0.477$ & $10.63 / \pm 0.424$ \\
\hline
\end{tabular}

\section{Conclusion}

To improve the isolation degree of the photoelectrical platform, in this paper, we analyzed the characteristic of perturbation and proposed a booting-type ADRC for the platform. The original model is modified by the equivalent disturbance voltage. Then, combining the customary square lead-lag controller, a strategy of active disturbance estimation and compensation is developed based on the booting-type ESO to generate the control value together.

Experimental results illustrated that the isolation degree is improved at least $7.12 \mathrm{~dB}$ at the working frequency. In higher frequency, the advantage of ADRC is more obvious. Besides, the ADRC is further robust to parameter changing. The isolation degree is still improved by ADRC with value changing of $15 \%$ in the parameter of the photoelectrical platform.

In summary, the booting-type ADRC improves the ability of disturbance rejection for the photoelectrical platform. This result is valuable in practical engineering. 


\section{Conflict of Interests}

The authors declare that there is no conflict of interests regarding the publication of this paper.

\section{Acknowledgment}

This work was supported in part by the National Science Foundation of China under Grant 61304032 and in part by the CIOMP Innovation Program under Grant Y3CXISS149.

\section{References}

[1] Z. Hurak and M. Rezac, "Image-based pointing and tracking for inertially stabilized airborne camera platform," IEEE Transactions on Control Systems Technology, vol. 20, no. 5, pp. 1146-1159, 2012.

[2] H. Xin and B. Li, "Optical orientation and shifting of a single multiwalled carbon nanotube," Light: Science \& Applications, vol. 3, p. e205, 2014.

[3] M. K. Masten, "Inertially stabilized platforms for optical imaging systems: tracking dynamic targets with mobile sensors," IEEE Control Systems Magazine, vol. 28, no. 1, pp. 47-64, 2008.

[4] J. M. Hilkert, "Inertially stabilized platform technology: concepts and principles," IEEE Control Systems Magazine, vol. 28, no. 1, pp. 26-46, 2008.

[5] H. Du and S. S. Nair, "Modeling and compensation of lowvelocity friction with bounds," IEEE Transactions on Control Systems Technology, vol. 7, no. 1, pp. 110-121, 1999.

[6] M. Sun, Z. Wang, Y. Wang, and Z. Chen, "On low-velocity compensation of brushless DC servo in the absence of friction model," IEEE Transactions on Industrial Electronics, vol. 60, no. 9, pp. 3897-3905, 2013.

[7] J. M. Hilkert, "A comparison of inertial line-of-sight stabilization techniques using mirrors," in 18th Acquisition, Tracking, and Pointing, vol. 5430 of Proceedings of SPIE, pp. 13-22, Orlando, Fla, USA, April 2004.

[8] S. Li and Z. Liu, "Adaptive speed control for permanent-magnet synchronous motor system with variations of load inertia," IEEE Transactions on Industrial Electronics, vol. 56, no. 8, pp. 30503059, 2009.

[9] Z. Q. Gao, "A paradigm shift in feedback control system design," in Proceedings of the American Control Conference, pp. 24512457, 2009.

[10] Z. Q. Gao, Y. Huang, and J. Q. Han, "An alternative paradigm for control system design," in Proceedings of the 40th IEEE Conference on Decision and Control (CDC'01), vol. 12, pp. 45784585, December 2001.

[11] H. Liu and S. Li, "Speed control for PMSM servo system using predictive functional control and extended state observer," IEEE Transactions on Industrial Electronics, vol. 59, no. 2, pp. 11711183, 2012.

[12] S. Li, J. Yang, W.-H. Chen, and X. Chen, "Generalized extended state observer based control for systems with mismatched uncertainties," IEEE Transactions on Industrial Electronics, vol. 59, no. 12, pp. 4792-4802, 2012.

[13] J. Yang, S. Li, and X. Yu, "Sliding-mode control for systems with mismatched uncertainties via a disturbance observer," IEEE
Transactions on Industrial Electronics, vol. 60, no. 1, pp. 160-169, 2013.

[14] Z. Q. Gao, "Scaling and bandwidth-parameterization based controller tuning," in Proceedings of the American Control Conference, vol. 6, pp. 4989-4996, Denver, Colo, USA, June 2003. 




Advances in

Operations Research

mansans

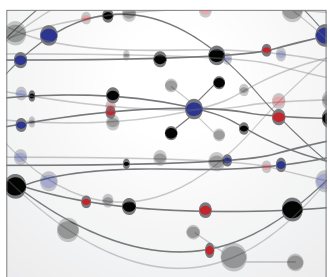

The Scientific World Journal



Submit your manuscripts at http://www.hindawi.com


Journal of

Function Spaces

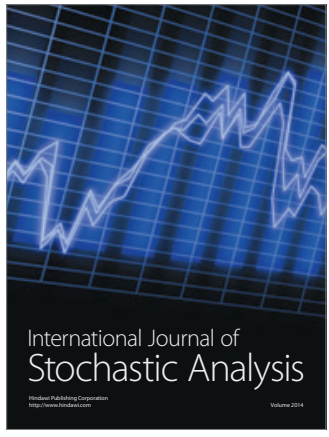

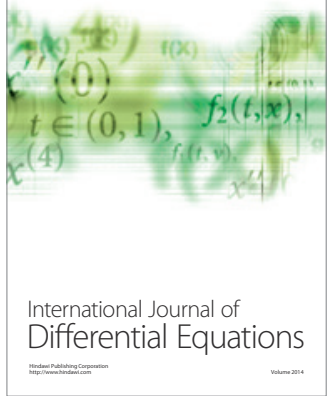
